\title{
Educação Positiva: A aplicação da Psicologia Positiva a instituições educacionais
}

\author{
Clarisse Lourenço Cintra \\ Universidade Federal do Espírito Santo - Vitória - ES - Brasil \\ Valeschka Martins Guerra \\ Universidade Federal do Espírito Santo - Vitória - ES - Brasil
}

\begin{abstract}
Resumo
A Educação Positiva pode ser definida, de forma ampla, como a aplicação da Psicologia Positiva a instituições de ensino. O início do movimento da Psicologia Positiva impulsionou a realização de estudos e práticas dedicados à promoção do bem-estar e ao funcionamento positivo de indivíduos, grupos e instituições. Nesse sentido, devido a seu papel crucial na sociedade, as instituições educacionais são consideradas locais privilegiados para promover o bem-estar, tanto na comunidade escolar como na sociedade em geral. Assim, a Educação Positiva tem por objetivo não só o desempenho acadêmico, mas também o florescimento dos estudantes e demais integrantes da comunidade escolar, preparando os jovens para se tornarem indivíduos realizados e cidadãos responsáveis. Levando em consideração a escassez de literatura sobre Educação Positiva no Brasil, este artigo pretende apresentar o desenvolvimento desta nova abordagem, resultante da interseção entre a Psicologia Positiva e o campo da educação, bem como seus aspectos teóricos e práticos.
\end{abstract}

Palavras-chave: Psicologia Positiva; Educação; Saúde mental.

\section{Positive Education: The application of Positive Psychology to educational institutions}

\begin{abstract}
The Positive Education can be broadly defined as the application of Positive Psychology to educational institutions. The beginning of the Positive Psychology movement led to the realization of studies and practices dedicated to the promotion of well-being and the positive functioning of individuals, groups and institutions. Because of that, due to their crucial role in society, educational institutions are considered privileged places to promote well-being, both in the school community and in the society in general. Thus, Positive Education aims not only at academic performance, but also at the flourishing of students and other members of the school community, in order to prepare young people to become accomplished individuals and responsible citizens. Taking into account the scarcity of literature on Positive Education in Brazil, this article intends to present the development of this new approach, resulting from the intersection between Positive Psychology and the field of education, as well as its theoretical and practical aspects.
\end{abstract}

Keywords: Positive Psychology; Education; Mental health.

\section{Educación Positiva: la aplicación de la Psicología Positiva a instituciones educacionales}

\section{Resumen}

Educación Positiva puede ser definida, de forma amplia, como la aplicación de la Psicología Positiva a instituciones de enseñanza. El inicio del movimiento de la Psicología Positiva impulsó la realización de estudios y prácticas dedicados a la promoción del bienestar y al funcionamiento positivo de individuos, grupos e instituciones. En ese sentido, debido a su papel fundamental en la sociedad, las instituciones educacionales son consideradas locales privilegiados para promover el bienestar, tanto en la comunidad escolar como en la sociedad en general. Así, la Educación Positiva tiene por objetivo no sólo el desempeño académico, sino también el florecimiento de los estudiantes y demás integrantes de la comunidad escolar, preparando los jóvenes para volverse individuos realizados y ciudadanos responsables. Llevando en consideración la escasez de literatura sobre Educación Positiva en Brasil, en este artículo se pretende presentar el desarrollo de este nuevo abordaje, resultante de la intersección entre la Psicología Positiva y el campo de la educación, así como sus aspectos teóricos y prácticos.

Palabras clave: Psicología Positiva; Educación; Salud mental. 


\section{Introdução}

Ao longo do tempo, o campo da educação tem sido foco de inúmeras investigações científicas, realizadas no Brasil e em todo o mundo, com o objetivo de compreender e avaliar suas práticas. Dessa forma, também buscam elaborar intervenções e métodos que possibilitem o aprimoramento dos sistemas educacionais (Pinto, Garcia, \& Letichevsky, 2006).

Grande parte dos estudos realizados nessa área se dedica, notadamente, a problemáticas relacionadas aos estudantes - como o insucesso escolar, dificuldades de aprendizagem, disfunções comportamentais e afetivas (e.g., bullying, depressão, ansiedade) - ou, por outro lado, relacionadas ao estresse, burnout e insatisfação profissional dos professores. Sendo assim, as pesquisas enfatizam, de modo geral, o adoecimento, as deficiências e o insucesso, ou seja, o que está 'dando errado' nos contextos educacionais (Pocinho \& Perestrelo, 2011; Rocha \& Sarriera, 2006).

No entanto, cada vez mais atenção vem sendo dedicada à compreensão e promoção do bom funcionamento dos indivíduos e instituições, oferecendo a contraparte necessária a uma visão mais integral do ser humano e da sociedade (Waters, 2011). Tal direcionamento foi impulsionado pelo movimento da Psicologia Positiva, que propõe o estudo do que 'dá certo', ou seja, das características humanas, sociais e institucionais que produzem bem-estar físico e psicológico (Pocinho \& Perestrelo, 2011). A abordagem da Psicologia Positiva busca, então, conhecer, elaborar, efetuar e avaliar meios que possibilitem o desenvolvimento e o funcionamento positivo dos indivíduos, grupos e instituições.

Nesse sentido, devido a seu papel crucial na sociedade, as instituições de ensino são locais privilegiados para a promoção de bem-estar não apenas da comunidade escolar (i.e., estudantes e seus familiares, professores e demais funcionários), mas da sociedade como um todo (Norrish, Williams, O'Connor, \& Robinson, 2013; Sun, 2013). Com base nesta perspectiva, a Educação Positiva desponta como um novo paradigma para a educação, a partir da aplicação da Psicologia Positiva a instituições educacionais, complementando as práticas tradicionais (Norrish \& cols., 2013). Considerando a escassez de literatura sobre o tema no Brasil e, de modo geral, em Língua Portuguesa (Cintra e Guerra, 2017), este é um artigo teórico que tem por objetivo apresentar o desenvolvimento dessa nova abordagem, seus aspectos teóricos e práticos, como a definição de Educação Positiva, suas características e exemplos de aplicação.

\section{Psicologia Positiva}

O ponto de partida do movimento denominado Psicologia Positiva se deu em 1997, quando Mihaly Csikszentmihalyi e Martin Seligman se encontraram e conversaram sobre como a psicologia estava focada essencialmente nos fenômenos patológicos. Ambos acreditavam que a ciência da experiência e do comportamento humanos deveria incluir, também, o estudo de seus aspetos positivos (Csikszentmihalyi, 2003).

Assim, ao assumir a presidência da American Psychological Association (APA), em 1998, Seligman propôs "uma nova ciência das forças humanas" (Seligman, 1999) e lançou, assim, as sementes da Psicologia Positiva. Nesse movimento, se convergiram os esforços e trabalhos de Seligman, Csikszentmihalyi e de outros estudiosos que buscam compreender e cultivar as forças e virtudes humanas, o bem-estar, a saúde mental e o funcionamento positivo (Peterson \& Park, 2003; Seligman, 1999).

Dando seguimento a essa iniciativa, uma edição especial da American Psychologist, dedicada exclusivamente à Psicologia Positiva, foi publicada em 2000. Nela, Seligman e Csikszentmihalyi (2000) introduziram o movimento com o artigo Positive Psychology: an introduction, muitas vezes considerado o ponto de partida oficial desta abordagem que propõe o estudo científico do funcionamento ótimo de indivíduos, grupos e instituições (Seligman \& Csikszentmihalyi, 2000; Gable \& Haidt, 2005).

No entanto, a Psicologia Positiva não pretende criticar as tradicionais correntes da psicologia ou negar a patologia, o sofrimento humano e os aspectos 'negativos' de suas experiências, características e instituições. Propõe, sim, que também seja abordado o outro lado, os seus aspectos positivos, proporcionando um equilíbrio na abordagem psicológica da experiência humana (Gable \& Haidt, 2005; Linley, Joseph, Harrington, \& Wood, 2006). Nas palavras de Seligman e Csikszentmihalyi (2000), "o objetivo da Psicologia Positiva é começar a catalisar uma mudança no foco da psicologia da preocupação somente com o reparo das piores coisas da vida para a construção, também, de qualidades positivas" (p. 5).

Considerada um termo 'guarda-chuva', que engloba teorias e pesquisas sobre emoções positivas, características individuais positivas e instituições positivas, sobre o que faz a vida valer a pena ser vivida (Noble \& McGrath, 2008; Seligman, Steen, Park \& Peterson, 2005), a Psicologia Positiva recebeu, desde seu surgimento, diversas definições (ver Linley \& cols., 2006; Hart \& Sasso, 2011). No entanto, apesar das diferenças, há consistências e temas centrais em torno dos quais o campo se desenvolve.

Desse modo, a Psicologia Positiva pode ser definida, de forma ampla, como o estudo científico das experiências e aspectos positivos do ser humano, suas potencialidades e motivações, e das condições que contribuem para a promoção do florescimento de indivíduos, grupos e instituições (Gable \& Haidt, 2005; Seligman \& Csikszentmihalyi, 2000). Além disso, com base na teoria do bem-estar, a Psicologia Positiva tem por principal objetivo "aumentar a quantidade de florescimento na vida das pessoas e do planeta" (Seligman, 2011, p.37), o que envolve a elevação nos níveis de emoções positivas, engajamento, propósito, realização, relacionamentos positivos, otimismo (Scorsolini-Comin, Fontaine, Koller, \& Santos, 2013; Seligman, 2011).

Florescimento (flourishing) se refere à experiência de que 'a vida está indo bem', uma combinação de sentir-se bem (componente hedônico) e funcionar de forma eficaz 
(componente eudaimônico), conotando um elevado nível de bem-estar psicológico, o que indica saúde mental. Assim, pode-se dizer que uma pessoa está florescendo quando percebe que sua vida está indo bem: ela está se sentindo bem (mais emoções positivas do que emoções negativas), funcionando bem (realização de seu potencial individual) e vivendo uma vida com propósito (fazer o bem) (Huppert \& So, 2009, 2013; ver também Fredrickson \& Losada, 2005).

Assim sendo, o florescimento inclui, fundamentalmente, os seguintes domínios: emoções positivas (alegria, gratidão), engajamento positivo (interesse, curiosidade), realização positiva (buscar e alcançar resultados significativos), propósito positivo (contribuir com a comunidade, ajudar outras pessoas) e relações positivas (habilidades sociais e emocionais) (ver Modelo PERMA, Seligman, 2011). Estes domínios e demais aspectos do florescimento (como resiliência, otimismo, flow) são alcançados de forma mais efetiva se sustentados pelo desenvolvimento das forças pessoais (i.e., forças de caráter dominantes em uma pessoa, ver Peterson \& Seligman, 2004) (Norrish \& cols., 2013). Estudos têm evidenciado que "indivíduos que estão florescendo (ou que têm um elevado nível de bem-estar psicológico), aprendem de forma eficaz, trabalham de maneira produtiva, têm melhores relações sociais, são mais propensos a contribuir com sua comunidade, e têm melhor saúde e expectativa de vida" (Huppert \& So, 2009, p. 1).

Ao se dedicar ao estudo dos aspectos saudáveis de seres humanos e comunidades, o movimento da Psicologia Positiva tem contribuído para uma visão mais equilibrada e completa da vivência humana, e para a compreensão de como e em que condições as emoções, características e instituições positivas promovem o florescimento. Nesse sentido, o desenvolvimento de estudos no campo da Psicologia Positiva continua avançando na direção de seu aprimoramento teórico, metodológico e prático, proporcionando, assim, diversas possibilidades de intervenções que, em diferentes contextos, ajudam na prevenção do adoecimento e na potencialização de aspectos positivos dos indivíduos e dos grupos. Entre esses contextos estão as instituições educacionais que, dada a sua importância para o desenvolvimento de indivíduos e sociedades, é um local privilegiado para a aplicação da Psicologia Positiva, por meio de intervenções que promovam o bem-estar e o florescimento de todos aqueles que participam da vida escolar. Este é, precisamente, o escopo da Educação Positiva.

\section{Educação Positiva}

O termo 'Educação Positiva' surgiu no início de 2008, durante um encontro entre Seligman e membros da equipe da Geelong Grammar School (GGS), escola australiana que foi a primeira no mundo a implementar os princípios da Psicologia Positiva em toda a instituição de ensino (Norrish, 2015). Na literatura, entretanto, o termo aparece pela primeira vez em 2009, no artigo Positive education: positive psychology and classroom interventions, de Seligman,
Ernst, Gillham, Reivich e Linkins (2009), no qual foi definida como educação tanto para as habilidades tradicionais como para a felicidade.

Desde então, outras definições (não opostas, mas complementares) foram atribuídas à Educação Positiva. Green, Oades e Robinson (2011) sugerem, numa definição mais ampla, que Educação Positiva é a "Psicologia Positiva aplicada à educação" (p. 16). White e Murray (2015), por sua vez, a definem como "um termo guarda-chuva utilizado para descrever intervenções empiricamente validadas e programas da Psicologia Positiva que têm um impacto no bem-estar do estudante" (p. 14). Para o diretor da GGS, Stephen Meek, "a definição de Educação Positiva é a união da ciência da Psicologia Positiva com a melhor prática de ensino, para estimular e apoiar o florescimento de escolas e indivíduos" (Norrish, 2015, p. 28).

Portanto, a Educação Positiva pode ser definida, de modo geral, como a Psicologia Positiva aplicada à educação (i.e., a instituições educacionais), preconizando que as habilidades para o bem-estar podem e devem ser ensinadas nas escolas junto às tradicionais habilidades para a qualificação e realização profissional (Green \& cols., 2011; Norrish \& cols., 2013; Waters, 2011). Desse modo, além de bem-estar, resiliência e florescimento, a Educação Positiva propicia caminhos para o que talvez seja um dos mais importantes aprendizados - o de conhecer a si mesmo (Norrish, 2015).

Ao longo das últimas gerações e no mundo inteiro, estudos apontam um crescimento dos índices de depressão, ansiedade, distúrbios alimentares, abuso de substâncias, entre outros problemas de saúde mental ou física, especialmente entre os jovens (Norrish, 2015; Seligman \& cols., 2009). Por outro lado, também são observadas inconsistências quanto aos níveis de felicidade (Seligman \& cols., 2009). Diante dessas questões, vale indagar se os modelos educacionais existentes estariam equipados e adequados para responder às demandas atuais, ou se há a necessidade de novas abordagens que possam fazer mais e melhor para ajudar os jovens a lidar com os desafios e prosperar em nossa complexa sociedade, além de experienciar mais propósito, realização, engajamento e relações significativas em suas vidas (Norrish, 2015).

As questões apresentadas acima já seriam razão suficiente para a inclusão do bem-estar na agenda educacional. No entanto, há outra boa razão para que o ensino de habilidades para o bem-estar passe a fazer parte dos currículos escolares: pesquisas revelam que aumentos no bem-estar tendem a melhorar também a aprendizagem e o rendimento acadêmico (Seligman \& cols., 2009). Outros estudos indicam que o bem-estar está relacionado à capacidade acadêmica, social e emocional e ao comportamento pró-social (Durlak, Weissberg, Dymnicki, Taylor, \& Schellinger, 2011). Além disso, intervenções em favor do bem-estar têm papel preventivo com relação à depressão, à ansiedade e ao estresse no ambiente escolar (Neil \& Christensen, 2007).

Assim, o bem-estar deveria "ser ensinado na escola por três motivos: como um antídoto à depressão, como um meio para aumentar a satisfação com a vida, e como um auxílio a 
uma melhor aprendizagem e a um pensamento mais criativo" (Seligman \& cols., 2009, p. 295). Nesse sentido, faz-se necessária uma revisão das metas educacionais, que devem passar a priorizar, além do aprendizado acadêmico tradicional, a educação social, emocional e ética, promovendo o bem-estar, as virtudes, e preparando os jovens para se tornarem indivíduos realizados e cidadãos responsáveis (Cohen, 2006).

Observamos, então, uma mudança de paradigma: as escolas passam a ser vistas como instituições cujo papel não se limita à formação em termos acadêmicos, mas se estende para desenvolver e preparar as crianças e os jovens como um todo (Huitt, 2010). Desse modo, as escolas não só oportunizam o florescimento psicológico, social e acadêmico de seus estudantes, como respondem aos crescentes índices de sofrimento psicológico e doença mental entre crianças e adolescentes, e à necessidade de abordar a questão da saúde mental de maneira mais proativa e preventiva (Green \& cols., 2011).

\section{Intervenções e programas}

No contexto educacional, iniciativas voltadas para o bem-estar podem ser observadas ao longo do tempo - como o foco na autoestima (anos 1970), nas habilidades sociais (anos 1990) e programas de resiliência (início do século 21). Em seguida, vemos programas contra o bullying, sobre valores, aprendizagem social e emocional, bem-estar do estudante - todos mostrando a mudança gradativa de uma abordagem focada apenas na deficiência e dificuldade, para uma que seja também mais positiva e preventiva (Green \& cols., 2011; Noble \& McGrath, 2008). Recentemente, este movimento tem sido impulsionado pelo surgimento da Educação Positiva (Green \& Norrish, 2013).

Inicialmente, os estudos e intervenções baseados na Psicologia Positiva se concentraram principalmente nas crianças e jovens estudantes. Assim, começaram a ser realizadas investigações sobre, por exemplo, a relação entre bem-estar e resultados acadêmicos, e foram colocados em prática programas como o Penn Resiliency Program e o Strath Haven Positive Psychology Curriculum (Cronlund, 2008; Norrish, 2015).

O Penn Resiliency Program (PRP), desenvolvido pelo Centro de Psicologia Positiva da Universidade da Pensilvânia (EUA), é um programa para grupos de adolescentes utilizado, desde 1990, em escolas de diferentes países. Os participantes recebem lições sobre habilidades cognitivo-comportamentais e sociais, com o objetivo de desenvolver resiliência, bem-estar e habilidades sociais e emocionais (Norrish, 2015).

O Strath Haven Positive Psychology Curriculum, por sua vez, consiste em um currículo, também desenvolvido pelo Centro de Psicologia Positiva da Universidade da Pensilvânia, para os estudantes da Strath Haven High School. O objetivo do programa, iniciado em 2003, é desenvolver as forças de caráter, relações de apoio, sentido de vida e, também, elevar as emoções positivas e reduzir as emoções negativas (Norrish, 2015).
Segundo Seligman e cols. (2009), sendo abordagens baseadas em evidências, esses dois programas fornecem suporte para o ensino do bem-estar nas escolas. O PRP, por exemplo, é um dos programas de prevenção da depressão em jovens que mais foi objeto de pesquisas. Durante os últimos 20 anos, 21 estudos avaliaram e indicaram sua eficácia na redução e prevenção da depressão e da ansiedade, redução do sentimento de impotência e aumento do otimismo e do bem-estar, e, ainda, redução dos problemas de conduta e melhora no comportamento relacionado à saúde (Seligman, 2011).

Além dos apresentados acima, outros programas de Educação Positiva são acompanhados por pesquisas, se destacam e servem de referencial, como o Bounce Back! (ver McGrath \& Noble, 2003), o programa Making Hope Happen for Kids (ver Lopez \& cols., 2004), o Strengths Gym (ver Proctor \& cols., 2011), o PROSPER (ver Noble \& McGrath, 2015) e, também, programas que implementam práticas contemplativas - como a atenção plena (mindfulness) - nas escolas (ver Huppert \& Johnson, 2010).

No que diz respeito à implementação de intervenções e programas de Psicologia Positiva nas escolas, esta pode se dar tanto de forma explícita, por meio de intervenções e programas estruturados, como implícita, por meio de práticas que empregam os princípios da Psicologia Positiva em diversos aspectos da instituição. A maior parte dos estudos já realizados sobre a aplicação da Psicologia Positiva em escolas aborda intervenções explícitas, as quais podem ter por foco o desenvolvimento de apenas um componente (e.g., gratidão) ou de vários conceitos da Psicologia Positiva, numa abordagem mais abrangente (Green \& Norrish, 2013).

Mais recentemente, vem sendo destacada a importância de implementar a Educação Positiva em toda a instituição, por meio de uma abordagem integral da escola (whole-school approach), que enfatiza a relevância de incorporar a Educação Positiva de forma ampla na comunidade escolar, para que se obtenha maior abrangência e sustentabilidade de seus resultados positivos (Sun, 2013; Waters, 2011). Essa abordagem ganhou destaque a partir do modelo criado na escola australiana Geelong Grammar School (GGS), o qual é considerado um importante referencial no campo da Educação Positiva, tanto por seu pioneirismo como pelo modo sistemático com que foi implementado e vem sendo acompanhado por avaliações e pesquisas, resultando numa vasta produção de manuscritos que serve de orientação para outras iniciativas, bem como de evidência da efetividade quanto aos resultados de sua aplicação. Por esse motivo, este modelo de aplicação será apresentado de forma mais detalhada a seguir.

Para alcançar seu propósito mais amplo - o de que todo o currículo e os relacionamentos estabelecidos na comunidade escolar tenham como base a geração e a manutenção do bem-estar -, esta abordagem integral da escola requer: (1) que os professores sejam equipados para ensinar diretamente a seus estudantes as habilidades da Psicologia Positiva; (2) que os princípios da Psicologia Positiva estejam integrados no currículo da escola; e (3) que os professores 
e demais funcionários sejam auxiliados a vivenciar os princípios da Psicologia Positiva (Cronlund, 2008).

Esse último ponto reflete o objetivo da Formação em Educação Positiva da GGS: fazer com que os professores tenham domínio das habilidades de bem-estar de forma mais pessoal, em vez de se concentrar apenas em prepará-los para transmitir esses conhecimentos a seus alunos (Cronlund, 2008). Desse modo, o treinamento de professores e funcionários os incentiva a uma vivência da Psicologia Positiva tanto na esfera profissional como na pessoal, promovendo seu bem-estar e florescimento.

É importante que professores e funcionários sintam, então, os efeitos do treinamento em Psicologia Positiva nas suas próprias vidas para que, assim, possam vivenciar e transmitir os princípios da Psicologia Positiva nas atividades e ações cotidianas, de forma genuína (Norrish, 2015). Além disso, "quando professores e funcionários da escola têm níveis elevados de bem-estar social e emocional, isso tem uma influência positiva nos estudantes" (Waters, 2011, p. 85), como tem ficado evidente em pesquisas que relacionam o bem-estar docente com o aprendizado e os resultados acadêmicos dos estudantes (Bajorek, Gulliford, \& Taskila, 2014; Bricheno, Brown, \& Lubansky, 2009).

Para arrematar essa abordagem extensiva, a GGS identificou como uma prioridade a inclusão de pais e familiares dos estudantes na implementação da Educação Positiva. Em 2013 foi realizado, então, o primeiro curso de Educação Positiva para pais e familiares, os quais podem, assim, apoiar o bem-estar de seus filhos em consonância com as ações da escola e, também, vivenciar seu próprio bem-estar (Norrish, 2015).

\section{O modelo aplicado da GGS}

O objetivo da Geelong Grammar School, desde o início de sua empreitada com a Psicologia Positiva, foi o de empregar a Educação Positiva de modo amplo, envolvendo toda a comunidade escolar. Tal proposta, que mudaria por completo a cultura de uma organização educacional tão grande como a GGS, era um empreendimento audacioso, e requeria planejamento à altura (Norrish, 2015).

Nesse sentido, "desenvolver uma estrutura de conceito e aplicação era visto como um passo essencial na incorporação da Educação Positiva em todos os níveis da comunidade escolar de forma sustentável e cientificamente embasada" (Norrish, 2015, p. 29). Para tanto, Robinson, Williams, Norrish e Brook reuniram-se, entre 2010 e 2012, para criar um modelo de Educação Positiva para a GGS (Norrish, 2015).

O Modelo Aplicado de Educação Positiva da GGS (GGS Applied Model for Positive Education) propõe que os seis domínios centrais do bem-estar - emoções positivas, engajamento positivo, realização positiva, propósito positivo, relacionamentos positivos e saúde positiva -, sejam cultivados, tendo por base as forças de caráter, para gerar florescimento (Norrish \& cols., 2013). Esses domínios são uma extensão e adaptação do modelo de florescimento PERMA (ver Seligman, 2011), diferindo deste notadamente por incluir a saúde positiva (Norrish, 2015).

O domínio das emoções positivas reconhece que a forma como estudantes, professores e demais membros da comunidade escolar se sentem tem papel importante no processo de ensino-aprendizagem, e traz benefícios sociais, físicos, pessoais e psicológicos. O objetivo é ajudá-los a

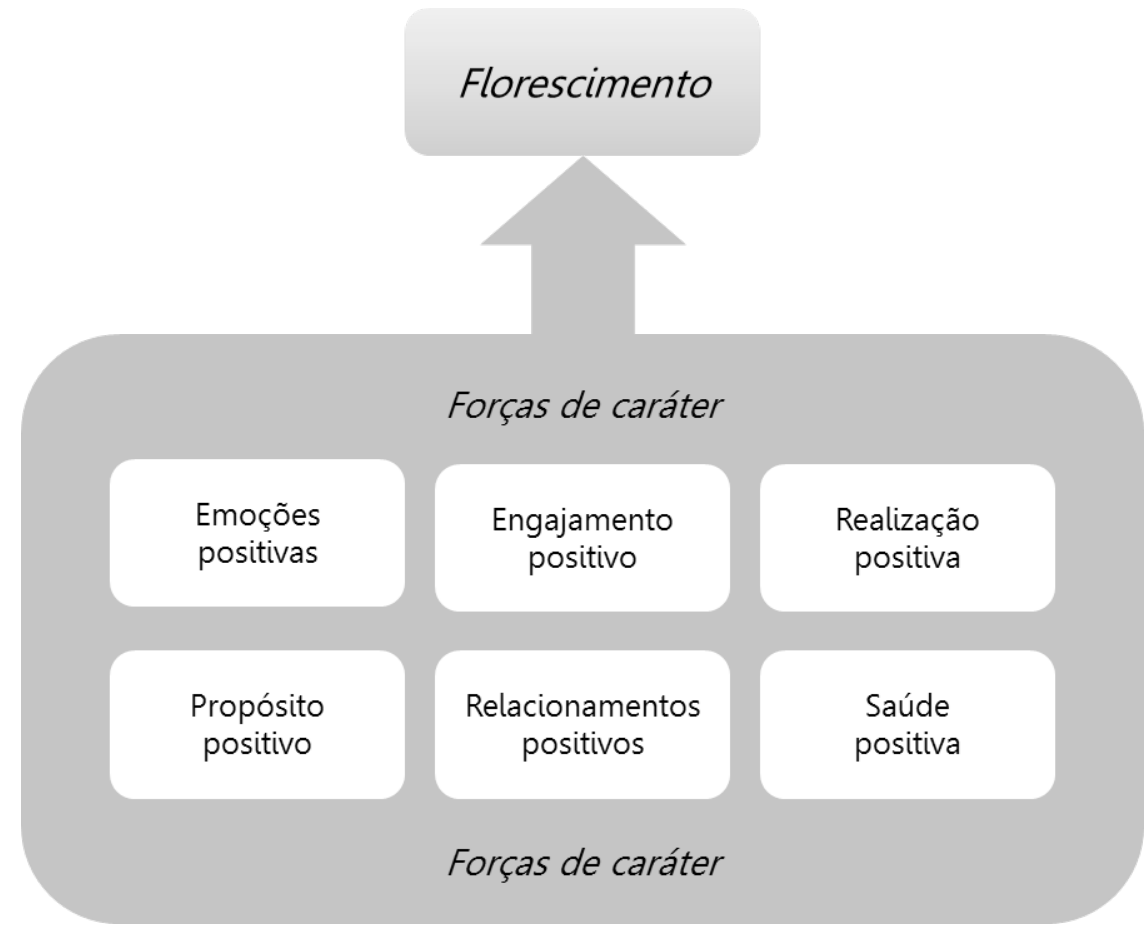

Figura 1. Modelo Aplicado de Educação Positiva da GGS. Fonte: Norrish, 2015. 
desenvolver uma compreensão das emoções (suas e dos outros), aceitar as emoções negativas ou desprazerosas (e.g., tristeza, ansiedade) como parte da vida, e cultivar a vivência de emoções positivas (e.g., alegria, esperança) em suas vidas diárias dentro e fora da escola (Norrish, 2015; Norrish \& cols., 2013).

O engajamento positivo é um domínio baseado no conceito de flow (ver Csikszentmihalyi, 1989) que envolve "ajudar estudantes e funcionários a experienciar uma completa imersão em atividades por meio da compreensão da natureza do engajamento, dos caminhos para alcançar tal imersão, e sua importância para o bem-estar" (Norrish, 2015, p. 33). Tal domínio trabalha para otimizar o processo de aprendizado e potencializar a motivação dentro e fora da escola (Norrish \& cols., 2013).

O domínio da realização positiva encoraja o estabelecimento e busca de metas, dentro e fora da vida escolar, que sejam recompensadoras para si e tragam benefícios para outras pessoas e para a comunidade como um todo. Assim, "desenvolve o potencial individual para buscar e alcançar resultados significativos" (Norrish, 2015, p. 33).

O propósito positivo está relacionado a um comprometimento com a comunidade e com a ajuda ao próximo, reconhecendo a importância de pertencer a uma comunidade escolar unida como fator protetivo para a saúde física e mental e para uma vida com significado e propósito. Este domínio envolve "compreender, acreditar e servir a algo maior do que si mesmo, e se engajar conscientemente em atividades para o benefício dos outros" (Norrish, 2015, p. 34), usando suas forças pessoais de forma altruísta.

O domínio dos relacionamentos positivos visa desenvolver habilidades emocionais e sociais que colaborem para o desenvolvimento de relações benéficas e significativas para si e para os outros, criando uma cultura de cuidado baseada em valores como bondade e perdão, e em uma comunicação atenta e respeitosa - active constructive responding (ver Gable \& Reis, 2010; Seligman, 2011). Tais fatores colaboraram para o aprofundamento e fortalecimento das relações entre todos os que participam do contexto escolar, favorecendo o sentimento de ligação com os outros e relações de apoio que são fundamentais para o bem-estar (Norrish, 2015; Norrish \& cols., 2013).

Por fim, o domínio da saúde positiva busca oferecer, aos membros da comunidade escolar, um conhecimento de base que os ajude a desenvolver hábitos que propiciem saúde física e psicológica (Norrish \& cols., 2013). Esses hábitos incluem comportamentos saudáveis como exercícios físicos, boa alimentação, e o desenvolvimento de habilidades como mindfulness (atenção plena) e resiliência (Norrish, 2015).

No modelo da GGS, estes seis domínios da Educação Positiva são propostos e integrados na escola em quatro níveis principais: aprender, viver, ensinar e integrar. Assim, professores e demais funcionários, junto com os familiares dos estudantes, aprendem sobre Psicologia Positiva em treinamentos regulares e são incentivados a viver os princípios da Educação Positiva e expressá-los em suas ações e interações entre si e com os estudantes. Ensinar refere-se ao aprendizado, pelos estudantes, da Educação Positiva de modo implícito (i.e., por meio da inserção dos conceitos de bem-estar no currículo mais amplo, abordando os objetivos acadêmicos de modo a também fomentar o florescimento) e explícito (i.e., em aulas sobre Educação Positiva, dedicadas diretamente ao cultivo do bem-estar e a encorajar e criar espaço para que os alunos reflitam sobre o significado e relevância dos conceitos aprendidos para suas vidas). Finalmente, integrar diz respeito à criação, de forma integrada (i.e., em toda a escola), de uma cultura e comunidade voltadas para o bem-estar (Norrish, 2015).

Nesse modelo de implementação, os princípios da Psicologia Positiva devem ser aplicados e integrados de forma contínua (i.e., um processo em constante evolução e desenvolvimento) em todo o currículo, cultura e aspectos da escola, para que despontem as condições favoráveis ao florescimento de todos que participam da comunidade escolar, e da própria instituição (Norrish, 2015; Waters, 2011). "Isso gera um clima de autenticidade, suporte estrutural e prática habitual, criando uma mudança normativa por meio da qual a Educação Positiva se torna autossustentável" (Sun, 2013, p. 3).

\section{Pesquisas e Avaliações}

Para compreender e avaliar que impactos e efeitos podem ser observados a partir da aplicação do modelo de Educação Positiva acima descrito, uma avaliação formal e independente, conduzida pela Universidade de Melbourne (Austrália), encontra-se em andamento. Paralelamente, alguns procedimentos vêm sendo realizados pela escola, tais como recolher evidências e declarações pessoais, pedir retorno dos alunos e comparar os índices de bem-estar da comunidade escolar com a média da população geral australiana. Desse modo, é possível verificar em que áreas os estudantes e funcionários estão florescendo e em que áreas eles se beneficiariam de mais apoio (Norrish, 2015).

Em 2011, três anos após a implementação inicial da Psicologia Positiva na GGS, estudantes responderam a um questionário sobre bem-estar. $A$ análise dos resultados indicou que os estudantes da GGS apresentavam escores acima da média nacional australiana. Os resultados também mostraram que, apesar de exibir taxas abaixo das médias nacionais, alguns estudantes apresentavam certas dificuldades como sintomas de depressão, solidão e estresse. Tal fato evidencia, entre outras coisas, a importância de manter sempre a oferta de ajuda adicional e suporte, como a disponibilização de psicólogos e conselheiros, para ajudá-los a lidar com essas dificuldades, desenvolver resiliência e construir as condições para a vivência de mais bem-estar (Norrish, 2015).

Uma das avaliações independentes, iniciada em 2013 e coordenada por Dianne Vella-Brodrick, da Universidade de Melbourne, envolveu estudantes da GGS e um grupo controle composto por estudantes de outra escola sociodemograficamente equivalente. Resultados iniciais mostraram que estudantes da GGS experienciaram sig- 
nificativo aumento dos níveis de bem-estar durante o ano e diminuição dos sintomas de depressão e de ansiedade; os estudantes do grupo controle, entretanto, apresentaram declínio no bem-estar (Norrish, 2015; ver Vella-Brodrick, Richard, \& Chin, 2014).

Esses e outros estudos independentes, qualitativos e quantitativos, vêm sendo realizados com o objetivo de compor um quadro abrangente dos efeitos da Educação Positiva no bem-estar. Os programas de pesquisa em andamento fornecerão valiosas informações sobre como oportunizar o florescimento daqueles que compõem a comunidade escolar na GGS, que estratégias devem ser priorizadas e como elas podem ser mais efetivas, a influência que cada ação e intervenção tem no bem-estar, os benefícios e dificuldades encontrados (Norrish, 2015).

Dessa forma, além de fornecer um referencial para que outras instituições educacionais apliquem os princípios da Psicologia Positiva em suas organizações, essas pesquisas e avaliações contribuem para a construção do conhecimento científico sobre o papel do bem-estar na educação e, de forma mais ampla, na sociedade. "A esperança é que isso irá capacitar outras escolas que buscam aumentar o foco em ajudar os estudantes a viver vidas mais resilientes, conectadas e com propósito" (Norrish, 2015, p. 286) e estender esses benefícios a todos os demais integrantes da comunidade.

\section{Considerações finais}

Assim como a Psicologia Positiva, a Educação Positiva não é a 'criação' de algo totalmente novo. Trabalhos anteriores já abordavam práticas e conceitos que hoje fazem parte do tecido da Educação Positiva, como o trabalho de Maria Montessori (Kahn, 2000) e as iniciativas anteriormente citadas, apenas para citar alguns exemplos. A grande contribuição dada pelo movimento da Psicologia Positiva consiste na reunião dessas teorias e práticas em um mesmo campo - facilitando a existência de um vocabulário comum, a construção do conhecimento e o desenvolvimento de novas abordagens, entre outros benefícios - e a utilização dos métodos e técnicas científicos no estudo de seus temas, conferindo-lhe maior credibilidade na comunidade científica e possibilitando aplicações e intervenções baseadas em evidências.

Embora possamos observar um grande progresso nos últimos anos, muito ainda resta a ser feito e compreendido sobre a Psicologia Positiva (ver Rusk \& Waters, 2013) e, portanto, sobre a Educação Positiva. Ao contrário dos programas que visam unicamente tratar e prevenir o sofrimento psicológico, patologias e comportamentos de risco, intervenções de Psicologia Positiva no contexto escolar não foram, ainda, amplamente estudadas (Green \& Norrish, 2013).

Não obstante, essas "intervenções que objetivam a construção de capacidades e forças, em contrapartida a abordagens que visam aliviar problemas ou corrigir défices, são inerentemente atrativas aos profissionais da educação, devido a seu foco construtivo e holístico" (Green \& Norrish, 2013, p.213-214). Isto posto, é necessária uma maior e me-
Ihor proximidade entre pesquisas e intervenções, com a realização de mais estudos que abordem o tema de modo mais abrangente e profundo, sempre aliando teoria e prática, pois a Educação Positiva é, acima de tudo, uma ciência aplicada.

No Brasil, algumas intervenções e aplicações da Psicologia Positiva em contextos educacionais já podem ser identificadas. A psicóloga Miriam Rodrigues (2015), por exemplo, desenvolveu uma abordagem chamada Educação Emocional Positiva, e realiza capacitação de profissionais da área da educação e implementação de projetos psicoeducacionais para o bem-estar em escolas. Em uma escola localizada no município do Rio de Janeiro, a coordenadora pedagógica e psicóloga Maria Aparecida Mussi (comunicação pessoal, julho, 2016) aplica princípios e realiza intervenções da Psicologia Positiva na instituição. Outro exemplo é o projeto Educação em Valores, Desenvolvimento Humano e Cultura de Paz, iniciado pela Secretaria de Educação do Espírito Santo, que tem o objetivo de ajudar os estudantes a lidar com as emoções e melhorar a capacidade de concentração e, para tanto, faz uso da meditação e do desenvolvimento da inteligência emocional (Lyrio, 2015).

Entretanto, em uma revisão sistemática sobre Educação Positiva, realizada em 2015, Cintra e Guerra (2017) encontraram um único artigo publicado no Brasil sobre o tema, o qual não fazia, contudo, nenhuma referência ao termo 'Educação Positiva'. Ainda assim, são encontrados alguns estudos que abordam conceitos da Psicologia Positiva relacionados ao campo da educação. Dentre eles, a maioria aborda pesquisas realizadas com professores ou estudantes, utilizando conceitos como sentido de vida, bem-estar, engajamento, forças de caráter (e.g. Cacciari, Guerra, Martins-Silva, Cintra, \& Castello, 2017; Damásio, Melo, \& Silva, 2013; Dela Coleta \& Dela Coleta, 2006; Pocinho \& Perestrelo, 2011).

Observamos que, como reflexo da relação muitas vezes distante entre as escolas e a academia, muitas das práticas e intervenções inovadoras não são acompanhadas por pesquisas científicas, notadamente no Brasil. Dessa forma, acabam por não serem estudadas e avaliadas e, com isso, além de não obterem dados sobre seus benefícios e eficácia, bem como outras informações valiosas para seu aprimoramento, ficam isoladas e restritas, pois não são compartilhadas de modo a fazerem parte da construção do conhecimento teórico e prático na área da educação.

Em vista disso, é necessário que as intervenções em escolas brasileiras sejam não só incentivadas, mas acompanhas por estudos que colaborem para seu desenvolvimento, aprimoramento e avaliação, e que seus resultados sejam divulgados. Dessa forma, se tornará possível a construção de conhecimentos e práticas de Educação Positiva adequadas e propícias ao contexto brasileiro.

A Educação Positiva não é, pois, um produto pronto a ser utilizado em qualquer instituição educacional, tampouco o Modelo Aplicado da GGS é a única forma de se colocar em prática a Educação Positiva, e outros referenciais podem ser identificados na literatura, como os programas e intervenções antes apresentados neste artigo, desenvolvidos, principalmente, na Austrália, Reino Unido e Estados Unidos 
- países onde as aplicações e pesquisas sobre Educação Positiva são mais difundidas. Nesse sentido, pesquisas longitudinais e que considerem diferentes culturas são necessárias, de modo geral, para que se possa compreender o impacto das intervenções e identificar as ações e atividades que têm maior efetividade na promoção do bem-estar e do florescimento nas diversas realidades socioculturais.

De acordo com os princípios da Educação Positiva, o sucesso educacional não mais é medido apenas pelo desempenho acadêmico dos estudantes e das instituições. Uma educação bem sucedida passa a ser assim considerada quando também promove o crescimento pessoal e o desenvolvimento positivo dos estudantes, como indivíduos e como cidadãos do mundo, abordando a existência humana de forma integral.

Finalmente, vale nos perguntarmos: o que é importante na e para a educação? Que tipo de instituições educacionais queremos criar para os profissionais, estudantes e demais membros da comunidade? O que queremos para nossas crianças e jovens e para seu futuro? Que mudanças queremos ser e ver no mundo? Esperamos que a Educação Positiva possa nos ajudar a responder a esses questionamentos, e que o resultado seja uma mudança sociocultural progressiva que, começando nas escolas e demais instituições e práticas educacionais, vá além de seus muros e espalhe seus benefícios pelo mundo, contribuindo para a construção de sociedades que gerem florescimento.

\section{Referências}

Bajorek, Z., Gulliford, J., \& Taskila, T. (2014). Healthy teachers, higher marks? Establishing a link between teacher health \& wellbeing and student outcomes. London: The Work Foundation.

Bricheno, P., Brown, S., \& Lubansky, R. (2009). Teacher well-being: A review of the evidence. London: Teacher Support Network.

Cacciari, M.B., Guerra, V.M., Martins-Silva, P.O., Cintra, C.L., \& Castello, N. (2017). Percepções de professores universitários brasileiros sobre as virtudes mais valorizadas no exercício da docência. Psicologia Escolar e Educacional, 21 (2), 313-322.

Cintra, C.L. \& Guerra, V.M. (2017). Educação Positiva: Uma revisão sistemática da literatura. Manuscrito em preparação.

Cohen, J. (2006). Social, emotional, ethical, and academic education: creating a climate for learning, participation in democracy, and well-being. Harvard Educational Review, 76, 201-237.

Cronlund, K.J. (2008). Analysis of positive psychology training at Geelong Grammar School. University of Pennsylvania, Pennsylvania, EUA.

Csikszentmihalyi, M. (1989). Flow: The Psychology of Optimal Experience [Resenha]. Recuperado: 16 jan. 2016. Disponível: https://www.researchgate.net/publication/224927532_Flow_The_ Psychology_of_Optimal_Experience
Csikszentmihalyi, M. (2003). Legs or wings? A reply to R. S. Lazarus. Psychological Inquiry, 14, 113-115.

Damásio, B.F., Melo, R.L.P., \& Silva, J.P. (2013). Sentido de vida, bemestar psicológico e qualidade de vida em professores escolares. Paidéia (Ribeirão Preto), 23(54), 73-82.

Dela Coleta, J.A. \& Dela Coleta, M.F. (2006). Felicidade, bemestar subjetivo e comportamento acadêmico de estudantes universitários. Psicologia em Estudo, 11, 533-539.

Durlak, J., Weissberg, R., Dymnicki, A., Taylor, R., \& Schellinger, K. (2011). The impact of enhancing students' social and emotional learning: A meta-analysis of school-based universal interventions. Child Development, 82(1), 405-432.

Fredrickson, B.L. \& Losada, M.F. (2005). Positive affect and the complex dynamics of human flourishing. American Psychologist, 60(7), 678-686.

Gable, S. \& Haidt, J. (2005). What (and why) is positive psychology? Review of General Psychology, 9(2), 103-110.

Gable, S. \& Reis, H. (2010). Good News! Capitalizing on Positive Events in an Interpersonal Context. Em M. Zanna (Org.), Advances in Experimental Social Psychology (pp. 198-257). New York: Elsevier Press.

Green, S. \& Norrish, J. (2013). Enhancing Well-Being in Adolescentes: Positive Psychology and Coaching Psychology Interventions in Schools. Em C. Proctor \& P. Linley (Orgs.), Research, applications and interventions for children and adolescents: $A$ positive psychology perspective (pp. 211-222). New York: Springer.

Green, S., Oades, L., \& Robinson, P. (2011). Positive education: Creating flourishing students, staff and schools. In Psych, 33(2). Recuperado: 24 fev. 2015. Disponível: http://www.psychology.org. au/publications/inpsych/2011/april/green.

Hart, K. \& Sasso, T. (2011). Mapping the contours of contemporary positive psychology. Canadian Psychology, 52, 82-92.

Huitt, W. (2010). Analyzing paradigms used in education and schooling. Educational psychology interactive. Valdosta: Valdosta State University.

Huppert, F.A. \& Johnson, D.M. (2010). A controlled trial of mindfulness training in schools: The importance of practice for an impact on well-being. The Journal of Positive Psychology, 5(4), 264-274.

Huppert, F.A. \& So, T.C. (2009, julho). What percentage of people in Europe are flourishing and what characterizes them? Trabalho apresentado na IX ISQOLS Conference, Florença, Itália.

Huppert, F.A. \& So, T.C. (2013). Flourishing across Europe: Application of a new conceptual framework for defining well-being. Social Indicators Research, 110(3), 837-861. 
Kahn, D. (2000). Montessori's positive psychology: A lasting imprint. NAMTA Journal, 25, 1-5.

Linley, P.A., Joseph, S., Harrington, S., \& Wood, A. M. (2006). Positive psychology: Past, present, and (possible) future. Journal of Positive Psychology, 1(1), 3-16.

Lopez, S., Snyder, C., Magyar-Moe, J., Edwards, L., Pedrotti, J., Janowski, K., Turner, J., \& Pressgrove, C. (2004). Strategies for accentuating hope. Em P. Linley \& S. Joseph (Orgs.), Positive Psychology in Practice (pp. 388-403). Hoboken, New Jersey: John Wiley \& Sons.

Lyrio, E. (2015, Setembro). Escola também é lugar de meditar e ficar mais calmo. Gazeta Online. Recuperado: 16 jan. 2016. Disponível: http://www.gazetaonline.com.br/_conteudo/ 2015/09/noticias/ cidades/3907759-escola-tambem-e-lugar-de-meditar-e-ficarmais-calmo.html

McGrath, H. \& Noble, T. (2003). Bounce Back! Teacher's Handbook. Sydney, Australia: Pearson Education.

Neil, A.L. \& Christensen, H. (2007). Australian school-based prevention and early intervention programs for anxiety and depression: A systematic review. The Medical Journal of Australia, 186, 305-308.

Noble, T. \& McGrath, H. (2008). The positive educational practices framework: A tool for facilitating the work of educational psychologists in promoting pupil wellbeing. Educational and Child Psychology, 25, 119-134.

Noble, T. \& McGrath, H. (2015). PROSPER: A New Framework for Positive Education. Psychology of Well-Being, 5, 1-17.

Norrish, J. (2015). Positive education: The Geelong Grammar School journey. Oxford: Oxford University Press.

Norrish, J., Williams, O., O'Connor, M., \& Robinson, J. (2013). An applied framework for positive education. International Journal of Wellbeing, 3(2), 147-161.

Peterson, C. \& Park, N. (2003). Positive Psychology as the evenhanded positive psychologists views it. Psychological Inquiry, 14, 143-147.

Peterson, C. \& Seligman, M. (2004). Character strengths and virtues: A handbook and classification. Oxford: Oxford University Press.

Pinto, F.C.F., Garcia, V.C., \& Letichevsky, A.C. (2006). Pesquisa Nacional Qualidade na Educação: A escola pública na opinião dos pais. Ensaio: aval. pol. públ. educ., 14(53), 527-542.

Pocinho, M. \& Perestrelo, C. (2011). Um ensaio sobre burnout, engagement e estratégias de coping na profissão docente. Educação e Pesquisa, 37(3), 513-528.
Proctor, C., Tsukayama, E., Wood, A., Maltby, J., Fox-Eades, J., \& Linley, P. (2011) Strengths Gym: The impact of a character strengths-based intervention on the life satisfaction and well-being of adolescents. The Journal of Positive Psychology, 6(5), 377-388.

Rocha, K.B. \& Sarriera, J.C. (2006). Saúde percebida em professores universitários: gênero, religião e condições de trabalho. Psicologia Escolar e Educacional, 10(2).

Rodrigues, M. (2015). Educação emocional positiva: saber lidar com as emoções é uma importante lição. Novo Hamburgo: Sinopsys.

Rusk, R. \& Waters, L. (2013). Tracing the size, reach, impact and breadth of positive psychology. Journal of Positive Psychology, 8(3), 207-221.

Scorsolini-Comin, F., Fontaine, A.M.G.V., Koller, S.H., \& Santos, M.A. (2013) From authentic happiness to well-being: The flourishing of positive psychology. Psicologia: Reflexão e Crítica, 26(4), 663-670.

Seligman, M. (1999). The president's address. American Psychologist, 54, 559-562.

Seligman, M. (2011). Florescer: Uma nova compreensão sobre a natureza da felicidade e do bem-estar. Rio de Janeiro: Objetiva.

Seligman, M. \& Csikszentmihalyi, M. (2000) Positive psychology: An introduction. American Psychologist, 55, 5-14.

Seligman, M., Ernst, R.M., Gillham, J., Reivich, K., \& Linkins, M. (2009). Positive education: positive psychology and classroom interventions. Oxford Review of Education, 35(3), 293-311.

Seligman, M., Steen, T., Park, N., \& Peterson, C. (2005). Positive psychology progress: empirical validation of interventions. American Psychologist, 60(5), 410.

Sun, J. (2013). An analysis of the implementation of positive education at Geelong Grammar School. Recuperado: 24 fev. 2015. Disponível: https://www.academia.edu/5231504/An_ Analysis_ of_the_Implementation_of_Positive_Education_at_Geelong_ Grammar_School_2013_

Vella-Brodrick, D.A., Rickard, N.S., \& Chin, T-C. (2014). An Evaluation of Positive Education at Geelong Grammar School: A Snapshot of 2013. Relatório de Pesquisa, University Of Melbourne, Austrália.

Waters, L. (2011). A review of school-based positive psychology interventions. The Australian Educational and Developmental Psychologist, 28(2), 75-90.

White, M.A. \& Murray, A.S. (2015). Building a positive institution. Em White M.A. \& A.S. Murray (Orgs.), Evidence-based approaches to positive education in schools (pp. 1-26). Holanda: Springer. 
Recebido em: 01 de Agosto de 2016 Aprovado em: 27 de dezembro de 2016

Sobre as autoras

Clarisse Lourenço Cintra (clarisselc@gmail.com)

Mestra em Psicologia, Grupo de Estudos e Práticas em Psicologia Positiva. Universidade Federal do Espírito Santo. Vitória-ES- Brasil

Valeschka Martins Guerra (valeschkamartins@gmail.com)

Doutora em Psicologia,

Grupo de Estudos e Práticas em Psicologia Positiva. Universidade Federal do Espírito Santo. Vitória-ES-Brasil.

Trabalho derivado da dissertação de mestrado da primeira autora, sob orientação da segunda autora, contando com o apoio do CNPq, por meio de bolsa de mestrado. 